\title{
Disrupted White Matter Functional Connectivity With the Cerebral Cortex in Migraine Patients
}

\section{OPEN ACCESS}

Edited by:

Thomas T. Liu,

University of California, San Diego,

United States

Reviewed by:

Ting Xue,

Inner Mongolia University of Science and Technology, China

Xianghong Arakaki,

Huntington Medical Research

Institutes, United States

${ }^{*}$ Correspondence: Jian-Ren Liu

liujr021@sjtu.edu.cn

Xiaoxia Du

duxiaoxia@sus.edu.cn

Specialty section:

This article was submitted to Brain Imaging Methods, a section of the journal

Frontiers in Neuroscience

Received: 22 October 2021

Accepted: 23 December 2021

Published: 13 January 2022

Citation:

Qin Z, Liang H-B, Li M, HuY, Wu J, Qiao Y, Liu J-R and Du X (2022)

Disrupted White Matter Functional Connectivity With the Cerebral Cortex in Migraine Patients.

Front. Neurosci. 15:799854. doi: 10.3389/fnins.2021.799854

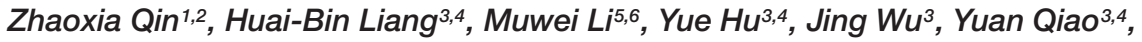 Jian-Ren Liu $^{3,4 *}$ and Xiaoxia Du ${ }^{1 *}$}

${ }^{1}$ School of Psychology, Shanghai University of Sport, Shanghai, China, ${ }^{2}$ Department of Medical Imaging, The Affiliated Hospital of Yangzhou University, Yangzhou, China, ${ }^{3}$ Department of Neurology, Jiuyuan Municipal Stroke Center, Shanghai Ninth People's Hospital, Shanghai Jiao Tong University School of Medicine, Shanghai, China, ${ }^{4}$ Clinical Research Center, Shanghai Jiao Tong University School of Medicine, Shanghai, China, ${ }^{5}$ Vanderbilt University Institute of Imaging Science, Nashville, TN, United States, ${ }^{6}$ Department of Radiology and Radiological Sciences, Vanderbilt University Medical Center, Nashville, TN, United States

Background: In attempts to understand the migraine patients' overall brain functional architecture, blood oxygenation level-dependent (BOLD) signals in the white matter (WM) and gray matter (GM) were considered in the current study. Migraine, a severe and multiphasic brain condition, is characterized by recurrent attacks of headaches. BOLD fluctuations in a resting state exhibit similar temporal and spectral profiles in both WM and GM. It is feasible to explore the functional interactions between WM tracts and GM regions in migraine.

Methods: Forty-eight migraineurs without aura (MWoA) and 48 healthy controls underwent resting-state functional magnetic resonance imaging. Pearson's correlations between the mean time courses of 48 white matter (WM) bundles and 82 gray matter (GM) regions were computed for each subject. Two-sample $t$-tests were performed on the Pearson's correlation coefficients (CC) to compare the differences between the MWOA and healthy controls in the GM-averaged CC of each bundle and the WM-averaged CC of each GM region.

Results: The MWoAs exhibited an overall decreased average temporal CC between BOLD signals in 82 GM regions and 48 WM bundles compared with healthy controls, while little was increased. In particular, WM bundles such as left anterior corona radiata, left external capsule and bilateral superior longitudinal fasciculus had significantly decreased mean CCs with GM in MWoA. On the other hand, 16 GM regions had significantly decreased mean CCs with WM in MWoA, including some areas that are parts of the somatosensory regions, auditory cortex, temporal areas, frontal areas, cingulate cortex, and parietal cortex.

Conclusion: Decreased functional connections between WM bundles and GM regions might contribute to disrupted functional connectivity between the parts of the pain processing pathway in MWoAs, which indicated that functional and connectivity abnormalities in cortical regions may not be limited to GM regions but are instead associated with functional abnormalities in WM tracts. 


\section{INTRODUCTION}

Migraine, a severe and multiphasic brain condition, is characterized by recurrent attacks of moderate-to-severe headache. The condition is usually accompanied by multiple sensory symptoms such as nausea, vomiting, and hypersensitivities to olfactory, visual, auditory, and somatosensory stimuli (Goadsby et al., 2017). Accumulated neuroimaging studies have found that migraine patients exhibit functional and structural abnormalities in a variety of cortical (Zhang et al., 2016, 2017a,b; Skorobogatykh et al., 2019), subcortical pain-processing regions (Qin et al., 2019, 2020a; Skorobogatykh et al., 2019; Younis et al., 2019; Kullmann and Veit, 2021) and white matter fiber tracts (Akatsuka et al., 2008; Ashina et al., 2021), that are often referred to as being part of the pain processing pathway. The pain processing pathway is believed to be involved in integrating all sensory, cognitive, and affective responses to pain, and it becomes active during nociceptive processing (Goadsby et al., 2017). Previous studies found white matter microstructure alterations in migraine patients by diffusion-tensor imaging (DTI) technology (Qin et al., 2019); however, function-related signals within the white matter (WM) tracts and functional interactions between the WM fibers and gray matter (GM) regions remain unclear.

Functional magnetic resonance imaging (fMRI) based on blood oxygenation level-dependent (BOLD) contrast has been widely used to detect changes in neural activity in the GM but can also identify similar meaningful signals in the WM (Gore et al., 2019). Considering the unique characteristics of white matter signals, some studies have observed reliable WM activation by using appropriate techniques (Mazerolle et al., 2013; Gawryluk et al., 2014). Recently, researchers have reported that BOLD signals in the WM convey rich functional information reflecting neural activity and connectivity, at rest or during tasks (Ding et al., 2016; Li et al., 2019). For example, resting-state and taskevoked magnetic resonance imaging (MRI) signals in the WM are modulated by neural activity in the GM tissues to which they connect (Wu et al., 2017). Ding et al. (2018) have recently shown that BOLD signal waveforms in stimulus-related WM pathways are synchronous with the applied stimulus, and the signal in the WM pathway shows apparent task specificity. Furthermore, the correlation of resting fMRI signals can detect WM bundle and specific GM functional areas, and these correlations are regulated by functional loading (Ding et al., 2018).

In attempts to understand the migraine patients' WM fibers and GM regions functional architecture, a novel method was used to investigate function-related signals within the WM tracts and functional interactions between the WM fibers and GM regions in the current study, and this method has been used in some brain diseases research (Gao et al., 2020; Huang et al., 2020; Lin et al., 2021). BOLD fluctuations in a resting state exhibit similar temporal and spectral profiles in both WM and GM, and their relatively low frequency $(0.01-0.1 \mathrm{~Hz})$ signal powers are comparable (Ding et al., 2016). Thus, it is feasible to explore the functional interactions between WM tracts and GM regions in migraine. In the current study, we used resting-state $\mathrm{fMRI}$ to explore the functional connectivity between WM fibers and GM regions in patients with migraines without aura (MWoA), which can illustrate the importance of WM brain function. We hypothesized that the resting-state functional connectivity between WM fibers and GM regions in patients with MWoA may change significantly; the altered functional connectivity may be associated with patients' clinical data such as headache frequency, pain intensity, duration of disease, headache impact test (HIT-6) score, and migraine disability assessment scale (MIDAS).

\section{MATERIALS AND METHODS}

\section{Subjects}

The Independent Ethics Committee approved this study of Shanghai Ninth People's Hospital [Project No. (2016) 01] and the Human Research Committee of East China Normal University (Project No. HR2015/03011). All procedures were performed following the Declaration of Helsinki, and all subjects signed written informed consent. Patients were diagnosed with MWoA by neurologists at the outpatient clinic of the Department of Neurology at Shanghai Ninth People's Hospital, according to the International Classification of Headache Disorders 3rd edition criteria (IHS, 2018). This study recruited 48 patients with MWoA (age: $38.1 \pm 10.4$ years) and 48 age and gender-matched healthy controls (HCs) (age: $39.0 \pm 11.0$ years) to perform the MRI experiments.

We also collected the age, gender, attack duration (hours), frequency (times/month), and pain intensity (VAS) of migraine patients. Patients also completed the MIDAS and HIT-6 to assess their headache-related disability accurately. Each patient filled out a questionnaire about the prevalence and traits of the headache, medications, and other diseases. None of the patients reported that they took preventive medicines or suffered from chronic migraines.

All patients with MWoA included in this study underwent an MRI scan during the interictal period. They had no headache 2 days before or 1 day after the MRI scan, and they did not have discomfort or migraine attacks during the MRI scan. The HCs had no headache or chronic pain disorder in the past year, and HCs with migraine previously and a family history of headaches were excluded. Also, immediate relatives of HCs did not have migraines or other headaches. Finally, the following exclusion criteria were followed for both the patient group and the control group: left-handedness, drug abuse, any neurological or psychiatric disease, metabolic disease (e.g., diabetes), or cardiovascular disease based on clinical examination and structured interviews. The demographic and clinical data are shown in Table 1.

\section{Magnetic Resonance Imaging Acquisition}

MRI imaging data were collected using a 3.0 Tesla Siemens Trio Tim system equipped with a 12-channel head coil. The subjects were instructed to stay still, close their eyes, and stay 
TABLE 1 | Demographic data and clinical scores of the MWoA group and control group.

\begin{tabular}{lccccc}
\hline & \multicolumn{2}{c}{ Migraine group } & & Control group & \\
\cline { 2 - 2 } & (Mean \pm SD) & & (Mean \pm SD) & P-value \\
\hline Gender (male) & 48 & & 48 & 1 \\
Age (years) & $29.2 \%$ & & $29.2 \%$ & 1 \\
Disease duration (years) & $38.1 \pm 10.4$ & & $39.0 \pm 11.0$ & 0.68 \\
Attack duration (hours) & $8.5 \pm 6.0$ & & - & - \\
Attack frequency (times/months) & $3.8 \pm 3.3$ & & - & - \\
Pain intensity VAS score & $7.2 \pm 1.8$ & & - & - \\
MIDAS score & $23.1 \pm 28.6$ & & - & - \\
HIT-6 score & $60.4 \pm 12.0$ & & - & - \\
\hline
\end{tabular}

VAS, visual analog scale; MIDAS, migraine disability assessment scale; HIT-6, headache impact test;-, No data The P-values are based on two-tailed t-tests.

awake and relaxed. During the scanning, all subjects wore Siemens earphones to reduce the impact of noise. The whole MRI scan lasted about $21 \mathrm{~min}$, including 2-3 min of adaptation and localization scan. The high-resolution T1-weighted images were acquired by a 3-dimensional magnetization-prepared rapid-acquisition gradient-echo pulse sequence. The main scanning parameters were as follows: echo time $=2.34 \mathrm{~ms}$, repetition time $=2,530 \mathrm{~ms}$, inversion time $=1,100 \mathrm{~ms}$, matrix size $=256 \times 256$, field of view $=256 \mathrm{~mm} \times 256 \mathrm{~mm}$, flip angle $=7^{\circ}$, number of slices $=192$, slice thickness $=1 \mathrm{~mm}$, sagittal orientation, scanning time: $6 \mathrm{~min} 3 \mathrm{~s}$. The high-resolution T2-weighted images were acquired by SPACE sequence. The main scanning parameters were as follows: echo time $=408 \mathrm{~ms}$, repetition time $=3,200 \mathrm{~ms}$, matrix size $=256 \times 256$, field of view $=256 \mathrm{~mm} \times 256 \mathrm{~mm}$, averages $=2$, number of slices $=192$, slice thickness $=1 \mathrm{~mm}$, sagittal orientation, scanning time: $4 \mathrm{~min} 59 \mathrm{~s}$. The images in the resting-state were generated by a T2*-weighted gradient-echo echo-planar imaging (EPI) pulse sequence. The parameters were as follows: transverse orientation; 210 volumes; flip angle, $90^{\circ}$; 33 slices of $3.5 \mathrm{~mm}$ thickness; repetition time, $2 \mathrm{~s}$; echo time, $30 \mathrm{~ms}$; the field of view, $220 \mathrm{~mm} \times 220 \mathrm{~mm}$; matrix size, $64 \times 64$; scanning time: $7 \mathrm{~min} 4 \mathrm{~s}$. There is no chemical reagent in the whole process, and the subjects can cooperate well. The noise of the general MRI scanning room is about $77.9 \mathrm{~dB}$. The EPI sequence is the loudest, about $110 \mathrm{~dB}$. The subjects were fitted with Siemens binaural active noise reduction headphones $(>10 \mathrm{~dB})$, and the noise was reduced to less than $100 \mathrm{~dB}$.

\section{Resting-State Data Preprocessing}

Data Processing Assistant for Resting-State fMRI (DPARSF) ${ }^{1}$ was used to preprocess resting-state fMRI data, and Statistical Parametric Mapping (SPM12) ${ }^{2}$ was used for functional data analysis. The first 10 volumes were discarded for signal equilibrium, and slice-timing was corrected. The images for each subject were motion-corrected, if the image's translation

\footnotetext{
${ }^{1}$ http://rfmri.org/DPARSF

${ }^{2}$ http://www.fil.ion.ucl.ac.uk/spm
}

was $>2 \mathrm{~mm}$ or their rotation was $>2^{\circ}$ in any direction have been discarded. We utilized the Friston 24-parameter model to regress out the head motion effects from the realignment. Then individual structural images were coregistered to the mean BOLD image. The transformed T1-weighted images were segmented into GM, WM, and cerebrospinal fluid regions and were normalized to the Montreal Neurological Institute space. Based on the same deformation field, EPI images were then normalized to the Montreal Neurological Institute (MNI) space using the Diffeomorphic Anatomical Registration Through Exponentiated Lie Algebra (DARTEL) tool. We performed temporal bandpass filtering $(0.01<\mathrm{f}<0.1 \mathrm{~Hz})$ to reduce the influence of lowfrequency drift and high-frequency respiratory and cardiac effects. Nuisance signals from the cerebrospinal fluid and linear trends were regressed out. The white-matter signals were not regressed out because this could eliminate signals of interest. Spatial smoothing was performed with a $4 \times 4 \times 4 \mathrm{~mm}^{3}$ full-width half-maximum Gaussian kernel. Finally, time series in the preprocessed BOLD images were normalized into unit variance voxelwise.

\section{Resting-State Correlations Between the White Matter and Gray Matter}

Resting-state correlation analyses were restricted to the GM and WM regions. According to Brodmann's definition, GM region masks were constructed by the PickAtlas tool (Maldjian et al., 2003), including 82 regions (41 cortical brain regions in each hemisphere). At the same time, WM tract masks from the JHU ICBM-DTI-81 WM atlas (Mori et al., 2008) were used to segment each subject into 48 bundle regions (21 bundles in each hemisphere and 6 commissure bundles). All bundle masks were further constrained within each subject's whole-brain WM mask with a high threshold of 0.8 to avoid signal contamination by nearby GM areas. Pearson's correlations between the mean time courses of each WM bundle and GM regions were computed for each subject. Fisher's $r$ to $z$ transformation was performed for further statistical analysis. These correlation coefficients were averaged among subjects in the group to obtain a group-level matrix representing the relationship between white matter and gray matter networks. The pipeline of fMRI data preprocessing and computing functional connections between each of the WM bundles and GM regions are presented in Supplementary Figure 1.

To visualize the differences in functional connections between migraine patients and healthy people, the average temporal correlations in a resting state between BOLD signals in $82 \mathrm{GM}$ regions and 48 WM bundles are shown in Figure 1, including the maps of average temporal correlations of the $\mathrm{HC}$ group or MWoA between BOLD signals, the maps of average temporal correlations of the $\mathrm{HC}$ minus the MWoAs or MWoA minus the HC between BOLD signals. Each element is the correlation coefficient (CC) between a pair of WM and GM regions averaged across each group. The abbreviations for the WM bundles are listed in Supplementary Table 1.

The two-sample $t$-test was conducted on the $\mathrm{z}$-score of the Pearson's CC to compare the differences between the MWoA 

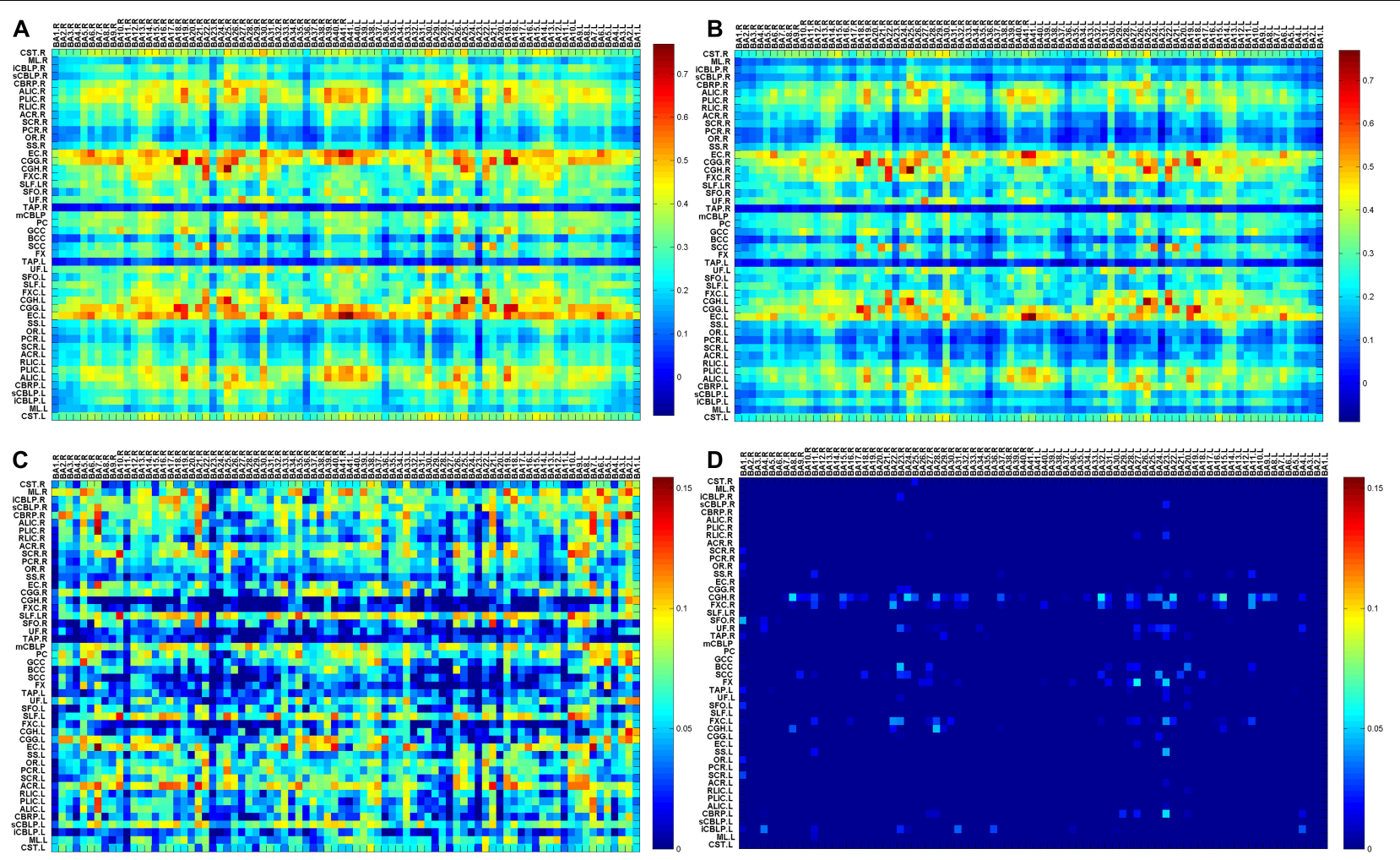

FIGURE 1 | (A) The map of average temporal correlations of the HC group between BOLD signals in 48 WM bundles and 82 GM regions; (B) the map of average temporal correlations of the MWoA group between BOLD signals in 48 WM bundles and 82 GM regions (C) the map of average temporal correlations of the HCs minus those of the patients with MWoA between BOLD signals in 48 WM bundles and 82 GM regions; (D) the map of average temporal correlations of the patients with MWoA minus those of the HCs between BOLD signals in 48 WM bundles and 82 GM regions. Each element is the correlation coefficient (CC) between a pair of WM and GM regions averaged across each group. BA denotes the Brodmann area, and abbreviations for the WM bundles are listed in Supplementary Table $\mathbf{1}$.

and HC groups in GM-averaged CC of each bundle and WMaveraged CC of each GM region. Two-sample $t$-tests were used to test the significance of the group differences with a false-positive correction $p<(1 / \mathrm{N})$ (regarding the WM-averaged CC of each bundle, $N=82$; regarding the GM-averaged CC of each WM region, $N=48$ ), which is equivalent to the expectation of less than one false-positive regional result per $48 \mathrm{WM}$ bundles or 82 GM regions at this threshold. False discovery rate correction (FDR) was used to correct for multiple comparisons. At the same time, $P<1 / \mathrm{N}$ for multiple testing corrections (Lynall et al., 2010; Fornito et al., 2011; Cao et al., 2021; Lin et al., 2021) were used to verify FDR results and explore the additional potential differences between the two groups.

\section{Correlations With Clinical Variables in Migraine}

Two sample $T$-test was used to interrogate demographic differences between MWoAs and healthy controls. Through the normal distribution test, it was found that the clinical data did not conform to the normal distribution, so Spearman correlation was used to analyze the clinical correlations of the MWoA group in GM-averaged CC of each bundle and WM-averaged CC of each GM region. Thresholds were $p<0.05$, and FDR was corrected for multiple comparisons.

\section{RESULTS}

\section{Demographic and Clinical Characteristics}

The demographic and clinical data of the migraine group and control group are shown in Table 1. There was no difference in age and gender between the two groups.

\section{Resting-State Correlations Between White Matter and Gray Matter}

We applied a false positive correction of FDR to determine the significance level between the two groups. And $p<1 / \mathrm{N}$ correction was used to verify and compare the results. There were 82 GM regions and 48 WM bundles; when analyzing GM-averaged correlation coefficients of WM bundles, $\mathrm{N}$ is 48 , $P<0.020833$ (Table 2); while when analyzing WM averaged correlation coefficients of GM bundles, $\mathrm{N}$ is $82, P<0.0 .012195$ (Table 3). Comparing $P<1 / \mathrm{N}$ and FDR correction, we found that $P<1 / \mathrm{N}$ correction had more positive results when $N=48$, and the results were more similar when $N=82$. For $P<1 / \mathrm{N}$ correction, the larger the $\mathrm{N}$, the greater the correction effect.

Figure 1 shows that most of the functional connections between the GM regions and the WM fibers were reduced in 
TABLE 2 | Summary of $t$-tests of GM-averaged correlation coefficients of WM bundles in migraine without aura (MWoA) compared to healthy controls (HC).

\begin{tabular}{|c|c|c|c|c|c|c|c|c|c|c|c|c|}
\hline \multirow[t]{3}{*}{ Bundle } & \multicolumn{5}{|c|}{ Right } & \multirow[b]{3}{*}{$P$ (FDR) } & \multicolumn{5}{|c|}{ Left } & \multirow[b]{3}{*}{$P$ (FDR) } \\
\hline & \multicolumn{2}{|c|}{ HC } & \multicolumn{2}{|c|}{ MWoA } & \multirow[b]{2}{*}{$\boldsymbol{P}$} & & \multicolumn{2}{|c|}{$\mathrm{HC}$} & \multicolumn{2}{|c|}{ MWoA } & \multirow[b]{2}{*}{$\boldsymbol{P}$} & \\
\hline & Mean & $S D$ & Mean & $S D$ & & & Mean & $S D$ & Mean & $S D$ & & \\
\hline Corticospinal tract & 0.358 & 0.151 & 0.310 & 0.151 & 0.137 & 0.193 & 0.349 & 0.157 & 0.293 & 0.152 & 0.081 & 0.134 \\
\hline Medial lemniscus & 0.194 & 0.149 & 0.12 & 0.150 & $0.016^{\star}$ & 0.081 & 0.192 & 0.161 & 0.136 & 0.137 & 0.063 & 0.125 \\
\hline Inferior cerebellar peduncle & 0.282 & 0.14 & 0.209 & 0.133 & $0.009^{\star}$ & 0.081 & 0.267 & 0.136 & 0.238 & 0.138 & 0.300 & 0.351 \\
\hline Superior cerebellar peduncle & 0.224 & 0.135 & 0.152 & 0.145 & $0.013^{\star}$ & 0.081 & 0.246 & 0.149 & 0.172 & 0.146 & $0.018^{\star}$ & 0.081 \\
\hline Cerebral peduncle & 0.366 & 0.127 & 0.299 & 0.154 & 0.031 & 0.096 & 0.351 & 0.136 & 0.314 & 0.141 & 0.213 & 0.269 \\
\hline Anterior limb of internal capsule & 0.385 & 0.143 & 0.325 & 0.145 & 0.042 & 0.096 & 0.374 & 0.145 & 0.325 & 0.151 & 0.086 & 0.137 \\
\hline Posterior limb of internal capsule & 0.398 & 0.138 & 0.340 & 0.154 & 0.047 & 0.103 & 0.389 & 0.141 & 0.338 & 0.149 & 0.079 & 0.134 \\
\hline Retrolenticular part of internal capsule & 0.306 & 0.142 & 0.263 & 0.126 & 0.093 & 0.137 & 0.276 & 0.153 & 0.226 & 0.122 & 0.065 & 0.125 \\
\hline Anterior corona radiata & 0.246 & 0.134 & 0.177 & 0.131 & $0.013^{*}$ & 0.081 & 0.248 & 0.125 & 0.163 & 0.129 & $0.002^{\star}$ & $0.048^{\#}$ \\
\hline Superior corona radiata & 0.235 & 0.146 & 0.167 & 0.145 & 0.022 & 0.081 & 0.227 & 0.141 & 0.169 & 0.146 & 0.051 & 0.106 \\
\hline Posterior corona radiata & 0.148 & 0.148 & 0.096 & 0.137 & 0.073 & 0.130 & 0.157 & 0.148 & 0.096 & 0.143 & 0.04 & 0.096 \\
\hline Posterior thalamic radiation (Include OR) & 0.15 & 0.152 & 0.102 & 0.134 & 0.094 & 0.137 & 0.195 & 0.135 & 0.132 & 0.125 & $0.020^{*}$ & 0.081 \\
\hline Sagittal stratum & 0.247 & 0.124 & 0.216 & 0.12 & 0.204 & 0.265 & 0.246 & 0.129 & 0.198 & 0.121 & 0.069 & 0.127 \\
\hline External capsule & 0.474 & 0.127 & 0.415 & 0.122 & 0.022 & 0.081 & 0.501 & 0.125 & 0.424 & 0.122 & $0.003^{\star}$ & $0.048^{\#}$ \\
\hline Cingulum (Cingulate gyrus) & 0.461 & 0.13 & 0.403 & 0.135 & 0.037 & 0.096 & 0.450 & 0.125 & 0.389 & 0.129 & 0.024 & 0.082 \\
\hline Cingulum (Hippocampus) & 0.36 & 0.135 & 0.357 & 0.12 & 0.947 & 0.947 & 0.387 & 0.145 & 0.345 & 0.134 & 0.176 & 0.235 \\
\hline Fornix (Cres)/Stria terminalis & 0.339 & 0.149 & 0.321 & 0.127 & 0.468 & 0.488 & 0.336 & 0.143 & 0.318 & 0.124 & 0.459 & 0.488 \\
\hline Superior longitudinal fasciculus & 0.293 & 0.14 & 0.209 & 0.129 & $0.004^{\star}$ & $0.048^{\#}$ & 0.311 & 0.132 & 0.233 & 0.125 & $0.004^{\star}$ & $0.048^{\#}$ \\
\hline Superior fronto-occipital fasciculus & 0.267 & 0.154 & 0.226 & 0.142 & 0.159 & 0.218 & 0.269 & 0.141 & 0.224 & 0.13 & 0.093 & 0.137 \\
\hline Uncinate fasciculus & 0.332 & 0.133 & 0.311 & 0.127 & 0.453 & 0.488 & 0.349 & 0.143 & 0.297 & 0.105 & 0.04 & 0.096 \\
\hline Tapetum & 0.02 & 0.181 & 0.003 & 0.125 & 0.538 & 0.549 & 0.061 & 0.168 & 0.028 & 0.142 & 0.282 & 0.338 \\
\hline \multirow[t]{2}{*}{ Bundle } & \multicolumn{5}{|c|}{$\mathrm{HC}$} & \multicolumn{5}{|c|}{ MWoA } & & \\
\hline & Mean & & SD & & & Mean & & SD & & $\mathbf{P}$ & & $\mathbf{P}$ (FDR) \\
\hline Middle cerebellar peduncle & 0.345 & & 0.156 & & & 0.268 & & 0.158 & & $0.015^{\star}$ & & 0.081 \\
\hline Pontine crossing tract (Part of MCP) & 0.325 & & 0.167 & & & 0.258 & & 0.15 & & 0.039 & & 0.096 \\
\hline Genu of corpus callosum & 0.318 & & 0.137 & & & 0.260 & & 0.129 & & 0.032 & & 0.096 \\
\hline Body of corpus callosum & 0.152 & & 0.161 & & & 0.118 & & 0.153 & & 0.275 & & 0.338 \\
\hline Splenium of corpus callosum & 0.278 & & 0.142 & & & 0.252 & & 0.140 & & 0.394 & & 0.450 \\
\hline Fornix (Column and body of fornix) & 0.261 & & 0.160 & & & 0.234 & & 0.164 & & 0.405 & & 0.452 \\
\hline
\end{tabular}

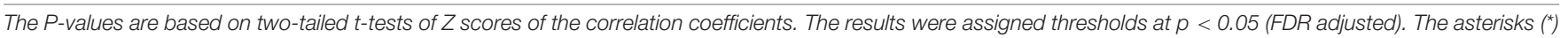

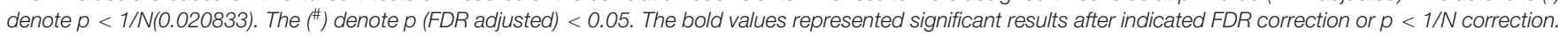

patients with MWoA relative to those in $\mathrm{HC}$, while little was increased. Quantitative comparisons between MWoA and HC in the mean CC of the GM regions across the WM bundles in the resting state are given in Figure 2 and Table 2. After FDR correction, left anterior corona radiata, left external capsule and bilateral superior longitudinal fasciculus had significantly reduced mean CCs with GM in MWoA. The WM bundles in the cerebrum of the MNI space with decreased GMaveraged CC after FDR correction are shown in Figure 3. While $p<1 / \mathrm{N}$ correction showed that other $5 \mathrm{WM}$ bundles had significantly decreased mean CCs with GM in patients with MWoA, including some tracts in the brainstem such as the right medial lemniscus, the right inferior cerebellar peduncle (ICP), the middle cerebellar peduncle (MCP), and the bilateral superior cerebellar peduncle (SCP), and some projection fibers such as the bilateral anterior corona radiate. In addition, association fibers such as the left posterior thalamic radiation (include optic radiation) were also affected.
Quantitative comparisons between MWoA and HC for the mean CC of the GM bundles across the WM regions at the resting state are given in Supplementary Figure 2 and Table 3. After FDR correction, they show that 16 GM regions had significantly decreased mean CCs with WM in patients with MWoA, such as sensorimotor regions including the left primary somatosensory cortex, the bilateral somatosensory association cortex, the bilateral visuomotor coordination, and the auditory cortex including the right auditory cortex, and frontal areas such as the bilateral anterior prefrontal cortex, the bilateral dorsolateral prefrontal cortex, the bilateral pars orbitalis, the left frontal eye fields, the left dorsolateral prefrontal cortex, the left pars triangularis, and the right middle temporal gyrus, and the cingulate cortex including the left ventral posterior cingulate cortex, the left ventral anterior cingulate cortex, the bilateral ectosplenial portion of the retrosplenial region, the left dorsal anterior cingulate cortex, and the left supramarginal gyrus. $P<1 / \mathrm{N}$ correction showed similar results with FDR, and only 
TABLE 3 | Summary of $t$-tests of WM-averaged correlation coefficients of GM bundles in migraine without aura (MWoA) compared to healthy controls (HC).

\begin{tabular}{|c|c|c|c|c|c|c|c|c|c|c|c|c|c|}
\hline \multicolumn{2}{|r|}{ Brodmann ROI } & \multicolumn{5}{|c|}{ Right } & \multirow[b]{3}{*}{$P$ (FDR) } & \multicolumn{5}{|c|}{ Left } & \multirow[b]{3}{*}{$\boldsymbol{P}$ (FDR) } \\
\hline & & \multicolumn{2}{|c|}{$\mathrm{HC}$} & \multicolumn{2}{|l|}{ MWoA } & \multirow[b]{2}{*}{$P$} & & \multicolumn{2}{|c|}{$\mathrm{HC}$} & \multicolumn{2}{|c|}{ MWoA } & \multirow[b]{2}{*}{$\boldsymbol{P}$} & \\
\hline & & Mean & $S D$ & Mean & $S D$ & & & Mean & $S D$ & Mean & $S D$ & & \\
\hline BA1 & Primary somatosensory cortex 1 & 0.193 & 0.138 & 0.175 & 0.144 & 0.466 & 0.484 & 0.185 & 0.144 & 0.126 & 0.135 & 0.035 & 0.074 \\
\hline $\mathrm{BA} 2$ & Primary somatosensory cortex2 & 0.239 & 0.143 & 0.177 & 0.161 & 0.040 & 0.076 & 0.254 & 0.125 & 0.170 & 0.140 & $0.003^{\star}$ & $0.038^{\#}$ \\
\hline BA3 & Primary somatosensory cortex 3 & 0.235 & 0.156 & 0.184 & 0.178 & 0.110 & 0.139 & 0.229 & 0.148 & 0.164 & 0.173 & 0.046 & 0.077 \\
\hline BA4 & Primary motor cortex & 0.251 & 0.158 & 0.219 & 0.163 & 0.291 & 0.310 & 0.244 & 0.161 & 0.213 & 0.159 & 0.284 & 0.306 \\
\hline BA5 & Somatosensory association cortex & 0.328 & 0.133 & 0.258 & 0.125 & $0.008^{\star}$ & $0.041^{\#}$ & 0.315 & 0.133 & 0.246 & 0.119 & $0.006^{\star}$ & $0.038^{\#}$ \\
\hline BA6 & Premotor and supplementary motor cortex & 0.306 & 0.156 & 0.251 & 0.165 & 0.079 & 0.112 & 0.308 & 0.148 & 0.255 & 0.159 & 0.088 & 0.122 \\
\hline BA7 & Visuo-motor coordination & 0.325 & 0.133 & 0.246 & 0.136 & $0.003^{\star}$ & $0.038^{\#}$ & 0.313 & 0.124 & 0.236 & 0.143 & $0.003^{\star}$ & $0.038^{\#}$ \\
\hline BA8 & Includes frontal eye fields & 0.280 & 0.141 & 0.234 & 0.100 & 0.039 & 0.076 & 0.304 & 0.129 & 0.229 & 0.108 & $0.002^{*}$ & 0.038 \\
\hline BA9 & Dorsolateral prefrontal cortex & 0.299 & 0.134 & 0.251 & 0.097 & 0.025 & 0.062 & 0.305 & 0.134 & 0.244 & 0.106 & $0.010^{\star}$ & $0.043^{\#}$ \\
\hline BA10 & Anterior prefrontal cortex & 0.335 & 0.109 & 0.273 & 0.104 & $0.004^{\star}$ & $0.038^{\#}$ & 0.331 & 0.113 & 0.266 & 0.114 & $0.006^{\star}$ & $0.038^{\#}$ \\
\hline BA11 & Orbitofrontal area & 0.257 & 0.117 & 0.234 & 0.112 & 0.229 & 0.254 & 0.262 & 0.126 & 0.237 & 0.117 & 0.224 & 0.254 \\
\hline BA17 & Primary visual cortex (V1) & 0.296 & 0.145 & 0.233 & 0.133 & 0.024 & 0.062 & 0.286 & 0.140 & 0.226 & 0.137 & 0.036 & 0.074 \\
\hline BA18 & Secondary visual cortex (V2) & 0.360 & 0.151 & 0.296 & 0.127 & 0.019 & 0.056 & 0.367 & 0.145 & 0.319 & 0.114 & 0.047 & 0.077 \\
\hline BA19 & Associative visual cortex (V3, V4, V5) & 0.378 & 0.139 & 0.319 & 0.132 & 0.022 & 0.060 & 0.356 & 0.141 & 0.309 & 0.123 & 0.051 & 0.077 \\
\hline BA20 & Inferior temporal gyrus & 0.332 & 0.126 & 0.284 & 0.137 & 0.051 & 0.077 & 0.335 & 0.131 & 0.293 & 0.136 & 0.097 & 0.124 \\
\hline BA21 & Middle temporal gyrus & 0.282 & 0.132 & 0.223 & 0.114 & 0.013 & $0.049^{\#}$ & 0.292 & 0.138 & 0.239 & 0.115 & 0.029 & 0.066 \\
\hline BA22 & Superior temporal gyrus & 0.267 & 0.160 & 0.202 & 0.126 & 0.020 & 0.057 & 0.261 & 0.150 & 0.210 & 0.123 & 0.047 & 0.077 \\
\hline BA23 & Ventral posterior cingulate cortex & 0.35 & 0.123 & 0.296 & 0.095 & 0.016 & 0.051 & 0.351 & 0.119 & 0.294 & 0.096 & $0.009^{\star}$ & $0.043^{\#}$ \\
\hline BA24 & Ventral anterior cingulate cortex & 0.367 & 0.130 & 0.310 & 0.113 & 0.019 & 0.056 & 0.367 & 0.129 & 0.300 & 0.113 & $0.008^{\star}$ & $0.041^{\#}$ \\
\hline BA25 & Subgenual area & 0.274 & 0.116 & 0.233 & 0.118 & 0.07 & 0.103 & 0.261 & 0.110 & 0.237 & 0.127 & 0.299 & 0.314 \\
\hline BA26 & Ectosplenial portion of the retrosplenial region & 0.325 & 0.125 & 0.257 & 0.128 & $0.006^{\star}$ & $0.038^{\#}$ & 0.329 & 0.117 & 0.263 & 0.116 & $0.005^{\star}$ & $0.038^{\#}$ \\
\hline BA27 & Piriform cortex & 0.352 & 0.136 & 0.294 & 0.122 & 0.029 & 0.066 & 0.318 & 0.128 & 0.266 & 0.108 & 0.03 & 0.067 \\
\hline BA28 & Ventral entorhinal cortex & 0.148 & 0.137 & 0.137 & 0.140 & 0.664 & 0.672 & 0.148 & 0.101 & 0.139 & 0.133 & 0.677 & 0.677 \\
\hline BA29 & Retrosplenial cingulate cortex & 0.29 & 0.125 & 0.257 & 0.098 & 0.127 & 0.153 & 0.304 & 0.123 & 0.269 & 0.102 & 0.125 & 0.153 \\
\hline ВАЗО & Part of cingulate cortex & 0.367 & 0.133 & 0.317 & 0.110 & 0.039 & 0.076 & 0.361 & 0.132 & 0.307 & 0.118 & 0.041 & 0.076 \\
\hline BA32 & Dorsal anterior cingulate cortex & 0.347 & 0.129 & 0.298 & 0.103 & 0.026 & 0.063 & 0.354 & 0.129 & 0.291 & 0.111 & $0.010^{\star}$ & $0.043^{\#}$ \\
\hline BA34 & Dorsal entorhinal cortex (Parahippocampal gyrus) & 0.295 & 0.137 & 0.267 & 0.118 & 0.229 & 0.254 & 0.257 & 0.134 & 0.241 & 0.121 & 0.484 & 0.496 \\
\hline BA35 & Perirhinal cortex & 0.239 & 0.145 & 0.200 & 0.131 & 0.155 & 0.182 & 0.225 & 0.129 & 0.195 & 0.128 & 0.247 & 0.270 \\
\hline BA36 & Ectorhinal area, now part of the perirhinal cortex & 0.249 & 0.135 & 0.206 & 0.135 & 0.093 & 0.123 & 0.259 & 0.142 & 0.220 & 0.134 & 0.127 & 0.153 \\
\hline BA37 & Fusiform gyrus & 0.404 & 0.135 & 0.361 & 0.124 & 0.076 & 0.109 & 0.385 & 0.139 & 0.345 & 0.130 & 0.089 & 0.122 \\
\hline BA38 & Temporopolar area & 0.268 & 0.128 & 0.220 & 0.120 & 0.046 & 0.077 & 0.249 & 0.123 & 0.210 & 0.116 & 0.093 & 0.123 \\
\hline BA39 & Angular gyrus, may be part of wernicke & 0.273 & 0.130 & 0.218 & 0.099 & 0.014 & 0.050 & 0.267 & 0.120 & 0.219 & 0.104 & 0.035 & 0.074 \\
\hline BA40 & Supramarginal gyrus, may be part of wernicke & 0.264 & 0.147 & 0.199 & 0.133 & 0.016 & 0.051 & 0.261 & 0.130 & 0.184 & 0.124 & $0.003^{\star}$ & $0.038^{\#}$ \\
\hline BA41 & Auditory cortex1 & 0.272 & 0.140 & 0.224 & 0.147 & 0.096 & 0.124 & 0.260 & 0.130 & 0.206 & 0.102 & 0.016 & 0.051 \\
\hline BA42 & Auditory cortex2 & 0.267 & 0.152 & 0.194 & 0.131 & $0.011^{*}$ & $0.045^{\#}$ & 0.259 & 0.143 & 0.209 & 0.120 & 0.047 & 0.077 \\
\hline BA43 & Primary gustatory cortex & 0.155 & 0.153 & 0.113 & 0.145 & 0.139 & 0.165 & 0.165 & 0.158 & 0.127 & 0.145 & 0.179 & 0.207 \\
\hline BA44 & Pars opercularis, part of IFG and part of broca & 0.230 & 0.148 & 0.179 & 0.133 & 0.051 & 0.077 & 0.249 & 0.139 & 0.178 & 0.139 & $0.012^{\star}$ & $0.047^{\#}$ \\
\hline BA45 & Pars triangularis, part of IFG and part of broca & 0.265 & 0.155 & 0.215 & 0.115 & 0.042 & 0.077 & 0.287 & 0.137 & 0.216 & 0.118 & $0.006^{\star}$ & $0.038^{\#}$ \\
\hline BA46 & Dorsolateral prefrontal cortex & 0.360 & 0.144 & 0.290 & 0.110 & $0.004^{\star}$ & $0.038^{\#}$ & 0.341 & 0.148 & 0.275 & 0.117 & $0.007^{\star}$ & $0.041^{\#}$ \\
\hline BA47 & Pars orbitalis, part of the inferior frontal gyrus & 0.312 & 0.139 & 0.247 & 0.101 & $0.005^{\star}$ & $0.038^{\#}$ & 0.297 & 0.132 & 0.246 & 0.110 & 0.025 & 0.062 \\
\hline BA48 & Retrosubicular area & 0.340 & 0.143 & 0.288 & 0.126 & 0.051 & 0.077 & 0.341 & 0.136 & 0.292 & 0.121 & 0.066 & 0.098 \\
\hline
\end{tabular}

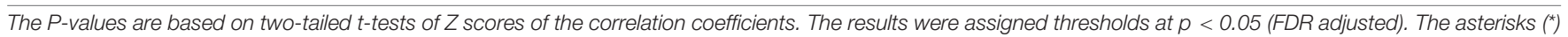
denote $p<N / 1$. The $\left(^{\#}\right)$ denote $p$ (FDR adjusted) $<0.05$. The bold values represented significant results after indicated FDR correction or $p<1 / N$ correction.

the right middle temporal gyrus pass FDR correction but did not pass $P<1 / \mathrm{N}$ correction. The GM regions in the cerebrum of the MNI space with decreased WM-averaged CCs are shown in Supplementary Figure 3.

\section{Correlations With Clinical Variables in Migraine}

We found a negative correlation between the patients' HIT- 6 score and the WM-averaged CC in the left anterior prefrontal cortex. The patient's pain intensity during headache attacks was negatively correlated with the GM-averaged CC in the middle cerebellar peduncle. A scatter plot of significant clinical relevance is shown in Figure 4. Detailed clinical correlation coefficients are given in Supplementary Tables 2, 3.

\section{DISCUSSION}

In the present study, we observed the functional connection between WM bundles and GM regions in MWoAs, which made up the gap between the previous studies of WM and GM. Patients 


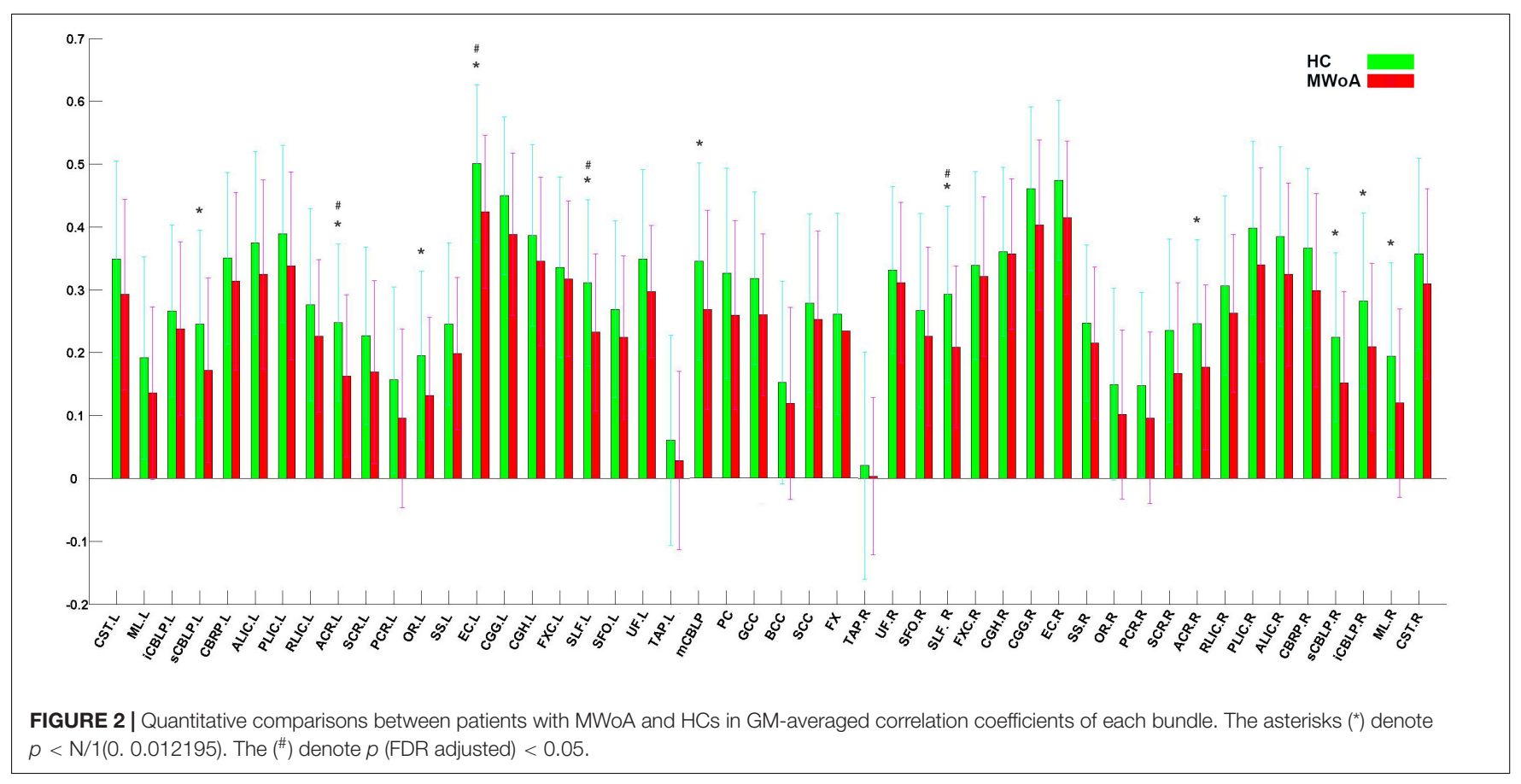

with MWoAs exhibited overall decreased average temporal CCs between BOLD signals in $48 \mathrm{WM}$ bundles and $82 \mathrm{GM}$ regions compared with HCs, while little was increased. In particular, after FDR correction left anterior corona radiata, left external capsule and bilateral superior longitudinal fasciculus had significantly decreased mean CCs with the GM in patients with MWoA. While $p<1 / \mathrm{N}$ correction showed that other 5 WM bundles had significantly decreased mean CCs with GM in patients with MWoA. These results are also discussed as exploratory results, revealing underlying WM functional change. The WM fibers

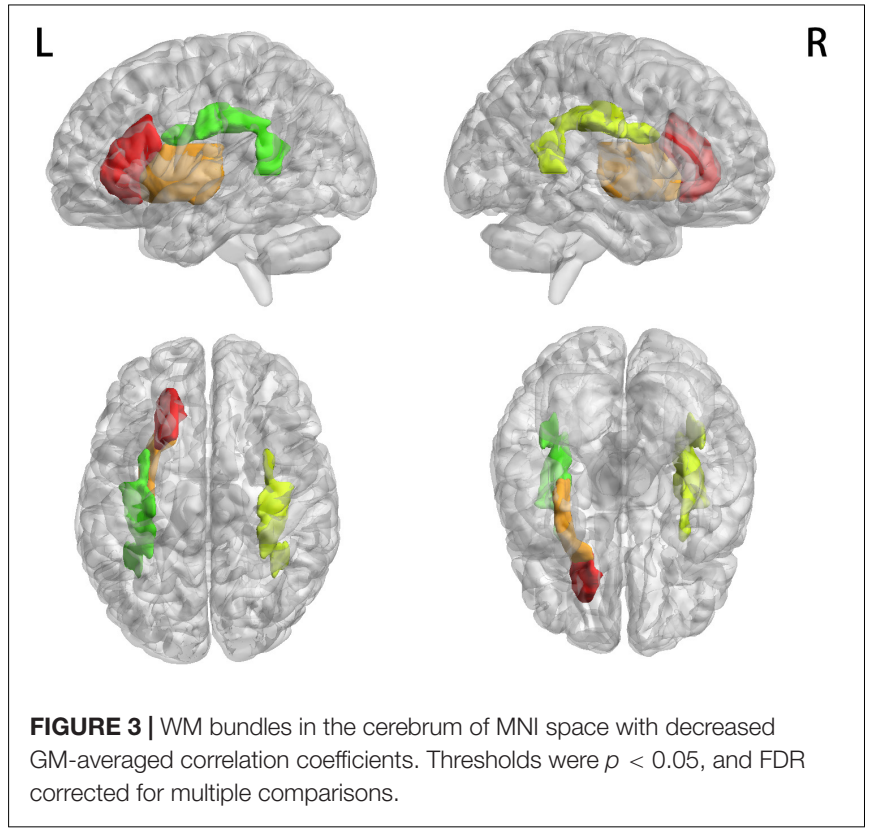

had decreased mean CCs with the GM in patients with MWoA, revealing functional alterations of the WM fibers. The functional alterations in the WM fibers might lead to a disruption in the transmission of signals between the nerve centers, eventually leading to dysfunction in the functional information integration between migraine-relevant centers.

\section{Projection Fibers}

Left anterior corona radiata had significantly decreased mean CC with GM, after FDR correction. Afferent and efferent projection fibers provide the connection between the cerebral cortex, brainstem, and spinal cord (Yakar et al., 2018). All of these fibers are distributed together as fan-shaped structures in a hemisphere and form the corona radiata (Yakar et al., 2018). The corona radiata is divided into three regions: anterior corona radiata, superior corona radiata, and posterior corona radiata. This region includes the thalamic radiations (corticothalamic, thalamocortical fibers) and parts of the long corticofugal pathways, such as the corticopontine tracts, corticospinal tracts, and corticobulbar tracts. Previous studies have shown that migraine patients exhibited decreased FA of the corona radiata at the level of the lateral ventricle horn along the trigeminothalamic tract (DaSilva et al., 2007). Patients' decreased functional connections in the anterior corona radiata with GM regions might lead to deficits in functional information transfer between the cerebral cortex, brainstem, and spinal cord.

\section{Association Fibers}

After FDR correction, the bilateral SLF and left external capsule exhibited reduced mean CC with GM. The SLF is located in the dorsolateral regions of the corona radiata and connects the front and back of the cerebrum, including the frontal, parietal, 


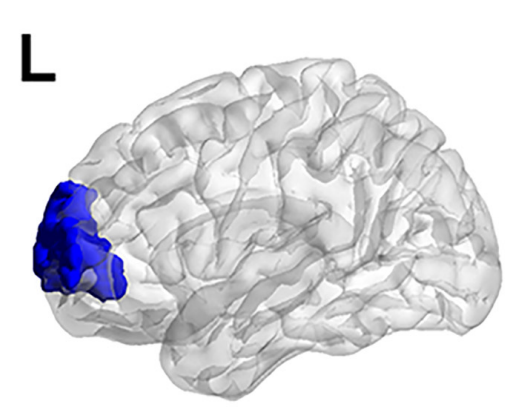

BA $10 \mathrm{~L}$

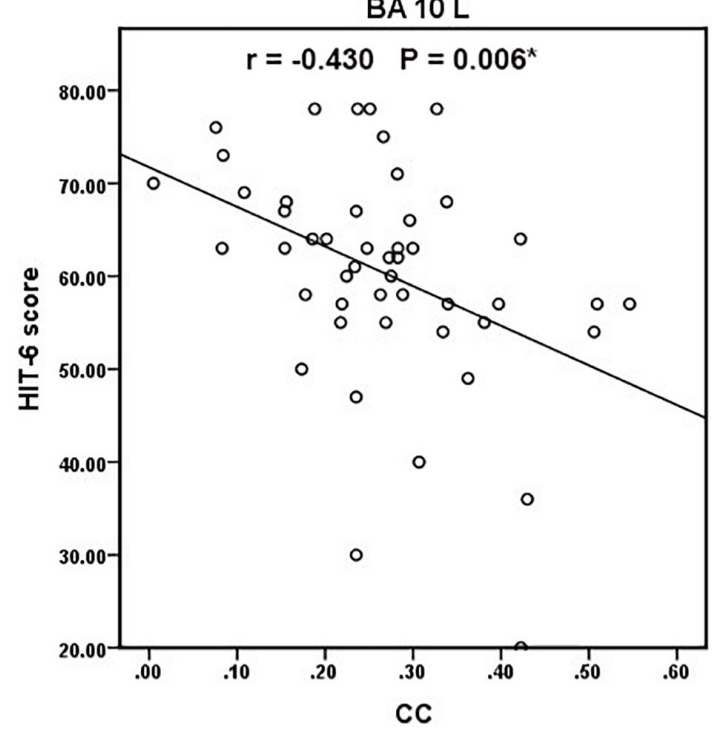

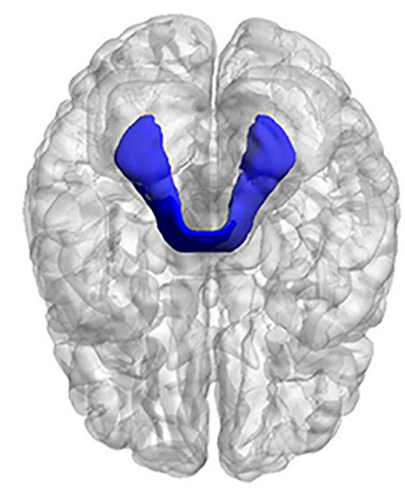

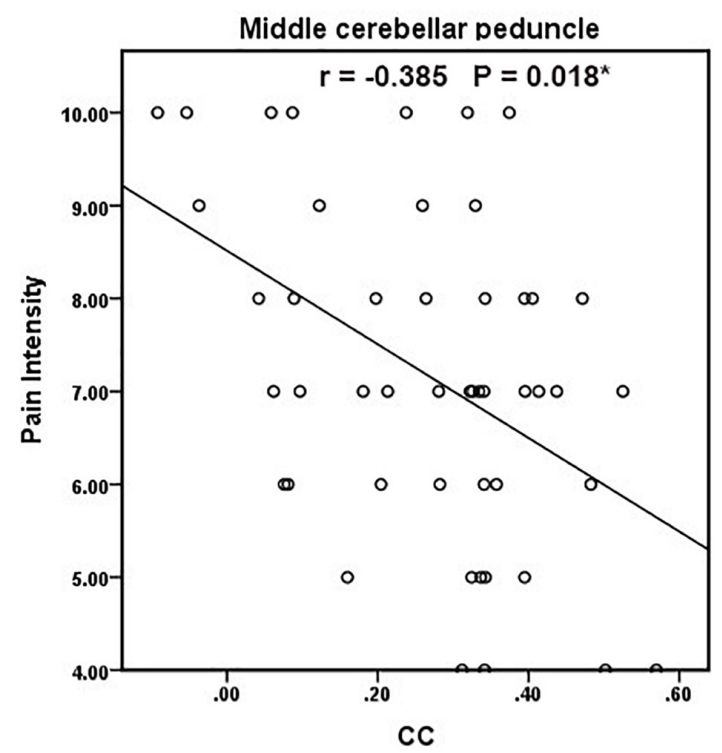

FIGURE 4 | Scatter plot of significant clinical correlations. The $\left(^{(}\right)$denote $p$ (FDR adjusted) $<0.05$.

occipital, and temporal lobes (Leung et al., 2018). SLF deficits in connecting these brain regions can affect the effectiveness of the prefrontal cortex in modulating the abovementioned multiple aspects of sensory or pain perception and can eventually lead to the development of headaches. The external capsule is located lateral to the internal capsule and is thought to contain association fibers, such as the SLF and inferior fronto-occipital fasciculus and commissural fibers (Mori et al., 2008). The extreme and external capsules lie in an anteroposterior disposition and are connected with the insula's anteroinferior part (Ribas et al., 2018). The lentiform nucleus is located between the internal and external capsules (Ribas et al., 2018). The external, internal, and extreme capsules connect the insular surface, basal ganglia, and thalamus to the cerebral lobes (Ribas et al., 2018). Thus, the external capsule is involved in anteroposterior connections and connections between subcortical and cortical regions relevant to migraine pathogenesis. Our results showed left external capsule decreased functional connectivity with GM in patients with MWoA, which might lead to impaired functional integration between numerous brain regions and may be related to patients' recurrent headaches and multiple sensory symptoms.
After $P<1 / \mathrm{N}$ correction, we found that patients with MWoA exhibited decreased GM-averaged CCs of the left posterior thalamic radiation (including optic radiation) and that this functional alteration might contribute to deficits in thalamic relay and modulation of the motor, sensory, and pain information, as well as dysfunction in visual information processing. Posterior thalamic radiation includes the corticothalamic and thalamocortical fibers and contains optic radiation (Mori et al., 2008). Thalamocortical activity plays an important role in abnormal sensory processing, which is a central feature of migraine attacks and could be used as a therapeutic target for pharmacological and neuromodulatory methods (such as transcranial magnetic stimulation) (Andreou et al., 2016). The thalamus's primary function is to transmit and regulate motor and sensory information, including pain modulation between numerous cortical regions and the peripheral nervous system (Herrero et al., 2002). Our previous study found that patients with MWoA exhibited a diminished level of functional connectivity between the subregions of the thalamus and left precuneus, right inferior parietal lobule, and right middle frontal gyrus (Qin et al., 2020c). A visual task specifically induced a stronger degree of 
temporal coherence within the optic radiations compared with the resting state, as well as significant correlations between the optic radiations and multiple cortical visual networks (Marussich et al., 2017); thus, optic radiation provides rich visual processing information. Although the migraine patients in this study had no aura, they had no visual aura; we observed WM abnormalities in the visual area. It is controversial whether there is an essential difference between migraine with aura and migraine without aura (Vgontzas and Burch, 2018). Migraine patients (both with and without aura) may be related to the abnormal integration of somatosensory, visual, auditory, and olfactory stimuli (Schwedt, 2013). Migraine patients with aura are explicitly related to visual hypersensitivity (Pearl et al., 2020). We speculate migraine with and without aura both have abnormal integration of sensory information and pain processing. Migraine with aura may be more sensitive to some kind of sensory sensitivity, such as visual sensitivity, and have a visual aura. In contrast, those who are not sensitive to visuals may have a "silent aura."

\section{Tracts in the Brainstem}

After $p<1 / \mathrm{N}$ correction, we found that right ICP, MCP, and bilateral SCP had decreased mean CCs with the GM in MWoA. The cerebellar peduncles are major white matter tracts of the cerebellum that communicate information among the cerebral cortex, the spinal cord, and the cerebellum. The ICPs connect the spinal cord, medulla, and cerebellum, carrying a variety of input and output fibers, which is mainly related to the integration of proprioception sensory input with motor vestibular function (Mori et al., 2005).

The MCP is emitted from pontine nuclei and transmits information between the cortex and cerebellum (Mori et al., 2008). The SCP transmits information between the dentate nucleus and thalamus (Mori et al., 2008). Migraine patients have a lower SCP volume and migraineurs with lower heat pain thresholds have smaller SCPs (Chong et al., 2017). Our previous study investigated that MWoAs exhibited altered microstructure in the ICP tract (Qin et al., 2019). These WM functional alterations in cerebellar peduncle tracts may cut off the relay stations in the cerebrocerebellar circuitry, eventually leading to dysfunction of conduction and integration of sensory and motor information among the medulla, the cerebellum, and cerebral cortex. The pain intensity of the migraineurs was negatively correlated with the GM-averaged CC in the middle cerebellar peduncle. This result further indicates that the reduced GMaveraged CC in the middle cerebellar peduncle is closely related to pain intensity in MWoAs.

After $P<1 / \mathrm{N}$ correction, our results showed that patients with MWoA exhibited decreased GM-averaged CCs at the right medial lemniscus, and this functional abnormality might contribute to patients with MWoA suffering from altered sensory information processing. The medial lemniscus travels along the dorsal side of the midbrain and pons and turns sharply toward the brainstem's ventral side at the level of the medulla (Wakana et al., 2004). The medial lemniscus is the primary sensory pathway toward the thalamus (Mori et al., 2008). Diffusion-weighted images analysis showed that fractional anisotropy (FA) in the region of the medial lemniscus/ventral trigeminal thalamic tract was significantly elevated in migraineurs compared with controls over the entire migraine cycle (Marciszewski et al., 2019).

\section{Gray Matter Regions}

On the other hand, 16 GM regions had significantly decreased mean CCs with WM in patients with MWoA (16 GM regions after FDR correction, 15 GM regions after $P<1 / \mathrm{N}$ correction), including some areas that are part of the somatosensory regions, auditory cortex, frontal areas, temporal gyrus, cingulate cortex, and parietal cortex. In line with previous studies (Zhang et al., 2016, 2017a,b; Jia and Yu, 2017; Ashina et al., 2021; Kim et al., 2021), migraine as a complex brain disease involves functional abnormalities in multiple cortical regions. These GM regions are believed to play critical roles in the pathophysiology of migraines. Our previous study indicated disrupted functional connectivity between the thalamus, sensorimotor areas, posterior pons, and other migraine-relevant brain regions in MWoA patients (Qin et al., 2020a,b,c). We speculated that the disrupted functional connections of the cortical regions in migraine patients may be due to the abnormal functional coupling between GM regions and WM regions. For example, the sensorimotor cortex's altered interactions with WM areas might contribute to the sensorimotor functional abnormalities and disrupted FC. Thus, our results further suggest that functional abnormalities and connectivity abnormalities in cortical regions may not be limited to gray matter regions but may be associated with functional abnormalities in white matter fiber tracts. In addition, correlation analysis, we obtained significantly negative correlations between HIT-6 scores and WM-averaged CCs in the left anterior prefrontal cortex, which suggested that the decrease of CC is significantly related to the impact of recurrent migraine attacks on the quality of daily life.

\section{Limitation}

The current study has several limitations. Firstly, only 82 GM regions of the cortex were considered, while subcortical regions were not included in the analysis. The structure of the subcortical brain area is more complex, including neurons and a large number of nerve fibers. The functional connections between subcortical areas and WM bundles also need to be further studied. In addition, we found no significant difference between male patients and female patients, and gender was used as a covariate in the analysis. In this study, women are the majority; the gender differences need to be further investigated in the future.

\section{CONCLUSION}

The patients with MWoA showed overall disrupted functional connections between WM bundles and GM regions compared with HCs, which might contribute to disrupted functional connectivity of the pain processing pathway. WM fibers exhibited functional abnormalities in some tracts in the brainstem and in some projection and association fibers in patients with MWoA, which might lead to dysfunctions in the conduction 
and integration of multisensory and motor information between cortical regions relevant in migraine pathogenesis and to deficits in the modulation of multiple aspects of sensory or pain perception, as well as abnormalities in visual information processing. Moreover, GM regions that play critical roles in migraine pathology exhibited decreased functional connectivity with WM areas in patients with MWoA. Thus, in patients with MWoA, functional abnormalities and connectivity abnormalities in cortical regions may not be limited to gray matter regions and are associated with functional abnormalities in white matter fiber tracts. Pain sensitivity and patient quality of life are closely tied to abnormal functional interactions between WM and GM areas. These findings provide new insights into the neural basis of migraine pathology and will benefit the diagnosis and treatment of this disorder.

\section{DATA AVAILABILITY STATEMENT}

The raw data supporting the conclusions of this article will be made available by the authors, without undue reservation.

\section{ETHICS STATEMENT}

The studies involving human participants were reviewed and approved by the Independent Ethics Committee of Shanghai Ninth People's Hospital [Project No. (2016) 01] and the Human Research Committee of East China Normal University (Project No. HR2015/03011). The patients/participants provided their written informed consent to participate in this study.

\section{AUTHOR CONTRIBUTIONS}

ZQ analyzed the data and prepared the manuscript. H-BL was responsible for evaluating the clinical data of the subjects

\section{REFERENCES}

Akatsuka, K., Noguchi, Y., Harada, T., Sadato, N., and Kakigi, R. (2008). Neural codes for somatosensory two-point discrimination in inferior parietal lobule: an fMRI study. Neuroimage 40, 852-858. doi: 10.1016/j.neuroimage.2007.12.013

Andreou, A. P., Holland, P. R., Akerman, S., Summ, O., Fredrick, J., and Goadsby, P. J. (2016). Transcranial magnetic stimulation and potential cortical and trigeminothalamic mechanisms in migraine. Brain 139, 2002-2014.

Ashina, S., Bentivegna, E., Martelletti, P., and Eikermann-Haerter, K. (2021). Structural and functional brain changes in migraine. Pain Ther. 10, 211-223. doi: 10.1007/s40122-021-00240-5

Cao, R., Yang, X., Luo, J., Wang, P., Meng, F., Xia, M., et al. (2021). The effects of cognitive behavioral therapy on the whole brain structural connectome in unmedicated patients with obsessive-compulsive disorder. Prog. Neuropsychopharmacol. Biol. Psychiatry 104:110037. doi: 10.1016/j.pnpbp.2020. 110037

Chong, C. D., Plasencia, J. D., Frakes, D. H., and Schwedt, T. J. (2017). Structural alterations of the brainstem in migraine. NeuroImage Clin. 13, 223-227.

DaSilva, A. F., Granziera, C., Tuch, D. S., Snyder, J., Vincent, M., and Hadjikhani, N. (2007). Interictal alterations of the trigeminal somatosensory pathway and periaqueductal gray matter in migraine. Neuroreport 18, 301-305. doi: 10.1097/ WNR.0b013e32801776bb and conducting experiments. ML was responsible for guiding the analysis of fMRI data. YH assisted in the collection of clinical data and the implementation of experiments. JW and YQ participated in the collection of clinical data and the implementation of experiments. J-RL designed the study and reviewed the manuscript. XD designed the study and prepared, revised the manuscript. All authors read and approved the final manuscript.

\section{FUNDING}

This research was supported by grants from the National Natural Science Foundation of China (No. 81571658 to XD and No. 81271302 to J-RL), the Research Innovation Project from Shanghai Municipal Science and Technology Commission (No. 14JC1404300, to J-RL), a project from Shanghai Municipal Education Commission-Gaofeng Clinical Medicine Grant Support (No. 20161422 to J-RL), a Clinical Research Project from Shanghaijiao Tong University School of Medicine (No. DLY201614 to J-RL), and the Biomedicine Key Program from Shanghai Municipal Science and Technology Commission (No. 16411953100 to J-RL).

\section{ACKNOWLEDGMENTS}

We thank Zhaohua Ding from the Institute of Imaging Science at Vanderbilt University Medical Center for his guidance and help in the data processing.

\section{SUPPLEMENTARY MATERIAL}

The Supplementary Material for this article can be found online at: https://www.frontiersin.org/articles/10.3389/fnins. 2021.799854/full\#supplementary-material

Ding, Z., Huang, Y., Bailey, S. K., Gao, Y., Cutting, L. E., Rogers, B. P., et al. (2018). Detection of synchronous brain activity in white matter tracts at rest and under functional loading. Proc. Natl. Acad. Sci. USA 115, 595-600. doi: 10.1073/pnas.1711567115

Ding, Z., Xu, R., Bailey, S. K., Wu, T. L., Morgan, V. L., Cutting, L. E., et al. (2016). Visualizing functional pathways in the human brain using correlation tensors and magnetic resonance imaging. Magn. Reson. Imag. 34, 8-17. doi: 10.1016/j.mri.2015.10.003

Fornito, A., Yoon, J., Zalesky, A., Bullmore, E. T., and Carter, C. S. (2011). General and specific functional connectivity disturbances in first-episode schizophrenia during cognitive control performance. Biol. Psychiatry 70, 64-72. doi: 10.1016/ j.biopsych.2011.02.019

Gao, Y., Sengupta, A., Li, M., Zu, Z., Rogers, B. P., Anderson, A. W., et al. (2020). Functional connectivity of white matter as a biomarker of cognitive decline in Alzheimer's disease. PLoS One 15:e0240513. doi: 10.1371/journal.pone.0240513

Gawryluk, J. R., Mazerolle, E. L., and D’Arcy, R. C. (2014). Does functional MRI detect activation in white matter? A review of emerging evidence, issues, and future directions. Front. Neurosci. 8:239. doi: 10.3389/fnins.2014. 00239

Goadsby, P. J., Holland, P. R., Martins-Oliveira, M., Hoffmann, J., Schankin, C., and Akerman, S. (2017). Pathophysiology of migraine: a disorder of sensory processing. Physiol. Rev. 97, 553-622. 
Gore, J. C., Li, M., Gao, Y., Wu, T. L., Schilling, K. G., Huang, Y., et al. (2019). Functional MRI and resting state connectivity in white matter - a mini-review. Magn. Reson. Imag. 63, 1-11. doi: 10.1016/j.mri.2019.07.017

Herrero, M.-T., Barcia, C., and Navarro, J. (2002). Functional anatomy of thalamus and basal ganglia. Child. Nerv. Syst. 18, 386-404.

Huang, J., Li, M., Li, Q., Yang, Z., Xin, B., Qi, Z., et al. (2020). Altered functional connectivity in white and gray matter in patients with multiple sclerosis. Front. Hum. Neurosci. 14:563048. doi: 10.3389/fnhum.2020.563048

IHS (2018). Headache classification committee of the international headache society (ihs) the international classification of headache disorders, 3rd edition. Cephalalgia 38, 1-11. doi: 10.1177/0333102417738202

Jia, Z., and Yu, S. (2017). Grey matter alterations in migraine: a systematic review and meta-analysis. Neuroimage Clin. 14, 130-140. doi: 10.1016/j.nicl.2017.01. 019

Kim, S. K., Nikolova, S., and Schwedt, T. J. (2021). Structural aberrations of the brain associated with migraine: a narrative review. Headache 61, 1159-1179. doi: $10.1111 /$ head.14189

Kullmann, S., and Veit, R. (2021). Resting-state functional connectivity of the human hypothalamus. Handb. Clin. Neurol. 179, 113-124. doi: 10.1016/B9780-12-819975-6.00005-4

Leung, A., Yang, E., Lim, M., Metzger-Smith, V., Theilmann, R., Song, D., et al. (2018). Pain-related white matter tract abnormalities in mild traumatic brain injury patients with persistent headache. Mol. Pain 14:1744806918810297. doi: $10.1177 / 1744806918810297$

Li, M., Newton, A. T., Anderson, A. W., Ding, Z., and Gore, J. C. (2019). Characterization of the hemodynamic response function in white matter tracts for event-related fMRI. Nat. Commun. 10:1140. doi: 10.1038/s41467-01909076-2

Lin, H., Li, M., Zhan, Y., Lin, L., Yang, K., Hu, S., et al. (2021). Disrupted white matter functional connectivity in aMCI APOEepsilon 4 carriers: a restingstate study. Brain Imag. Behav. 15, 1739-1747. doi: 10.1007/s11682-020-00 367-7

Lynall, M. E., Bassett, D. S., Kerwin, R., McKenna, P. J., Kitzbichler, M., Muller, U., et al. (2010). Functional connectivity and brain networks in schizophrenia. J. Neurosci. 30, 9477-9487. doi: 10.1523/JNEUROSCI.0333-10.2010

Maldjian, J. A., Laurienti, P. J., Kraft, R. A., and Burdette, J. H. (2003). An automated method for neuroanatomic and cytoarchitectonic atlas-based interrogation of fMRI data sets. Neuroimage 19, 1233-1239. doi: 10.1016/s10538119(03)00169-1

Marciszewski, K. K., Meylakh, N., Di Pietro, F., Macefield, V. G., Macey, P. M., and Henderson, L. A. (2019). Fluctuating regional brainstem diffusion imaging measures of microstructure across the migraine cycle. eNeuro 6:2019. doi: 10 . 1523/ENEURO.0005-19.2019

Marussich, L., Lu, K. H., Wen, H., and Liu, Z. (2017). Mapping white-matter functional organization at rest and during naturalistic visual perception. Neuroimage 146, 1128-1141. doi: 10.1016/j.neuroimage.2016.10.005

Mazerolle, E. L., Gawryluk, J. R., Dillen, K. N., Patterson, S. A., Feindel, K. W., Beyea, S. D., et al. (2013). Sensitivity to white matter FMRI activation increases with field strength. PLoS One 8:e58130. doi: 10.1371/journal.pone.0058130

Mori, S., Oishi, K., Jiang, H., Jiang, L., Li, X., Akhter, K., et al. (2008). Stereotaxic white matter atlas based on diffusion tensor imaging in an ICBM template. Neuroimage 40, 570-582. doi: 10.1016/j.neuroimage.2007.12.035

Mori, S., Wakana, S., Van Zijl, P. C., and Nagae-Poetscher, L. (2005). MRI atlas of human white matter 1st Edition. Amsterdam: Elsevier.

Pearl, T. A., Dumkrieger, G., Chong, C. D., Dodick, D. W., and Schwedt, T. J. (2020). Sensory hypersensitivity symptoms in migraine with vs without aura: results from the american registry for migraine research. Headache 60, 506-514. doi: 10.1111/head.13745

Qin, Z., He, X. W., Zhang, J., Xu, S., Li, G. F., Su, J., et al. (2019). Structural changes of cerebellum and brainstem in migraine without aura. J. Headache Pain 20:93. doi: 10.1186/s10194-019-1045-5

Qin, Z., He, X. W., Zhang, J., Xu, S., Li, G. F., Su, J., et al. (2020a). Altered spontaneous activity and functional connectivity in the posterior pons of patients with migraine without aura. J. Pain 21, 347-354. doi: 10.1016/j.jpain. 2019.08.001

Qin, Z., Su, J., He, X. W., Ban, S., Zhu, Q., Cui, Y., et al. (2020b). Disrupted functional connectivity between sub-regions in the sensorimotor areas and cortex in migraine without aura. J. Headache Pain 21:47. doi: 10.1186/s10194020-01118-1

Qin, Z. X., Su, J. J., He, X. W., Zhu, Q., Cui, Y. Y., Zhang, J. L., et al. (2020c). Altered resting-state functional connectivity between subregions in the thalamus and cortex in migraine without aura. Eur. J. Neurol. 27, 2233-2241. doi: 10.1111/ ene. 14411

Ribas, E. C., Yağxmurlu, K., de Oliveira, E., Ribas, G. C., and Rhoton, A. (2018). Microsurgical anatomy of the central core of the brain. J. Neurosurg. 129, 752-769. doi: 10.3171/2017.5.jns162897

Schwedt, T. J. (2013). Multisensory integration in migraine. Curr. Opin. Neurol. 26, 248-253. doi: 10.1097/WCO.0b013e328360edb1

Skorobogatykh, K., van Hoogstraten, W. S., Degan, D., Prischepa, A., Savitskaya, A., Ileen, B. M., et al. (2019). Functional connectivity studies in migraine: what have we learned? J. Headache Pain 20:108. doi: 10.1186/s10194-019-1047-3

Vgontzas, A., and Burch, R. (2018). Episodic migraine with and without aura: key differences and implications for pathophysiology, management, and assessing risks. Curr. Pain Headache Rep. 22:78. doi: 10.1007/s11916-018-0735-z

Wakana, S., Jiang, H., Nagae-Poetscher, L. M., van Zijl, P. C., and Mori, S. (2004). Fiber tract-based atlas of human white matter anatomy. Radiology 230, 77-87. doi: 10.1148/radiol.2301021640

Wu, X., Yang, Z., Bailey, S. K., Zhou, J., Cutting, L. E., Gore, J. C., et al. (2017). Functional connectivity and activity of white matter in somatosensory pathways under tactile stimulations. Neuroimage 152, 371-380. doi: 10.1016/j. neuroimage.2017.02.074

Yakar, F., Eroglu, U., Peker, E., Armagan, E., Comert, A., and Ugur, H. C. (2018). Structure of corona radiata and tapetum fibers in ventricular surgery. J. Clin. Neurosci. 57, 143-148. doi: 10.1016/j.jocn.2018.08.041

Younis, S., Hougaard, A., Noseda, R., and Ashina, M. (2019). Current understanding of thalamic structure and function in migraine. Cephalalgia 39, 1675-1682. doi: 10.1177/0333102418791595

Zhang, J., Su, J., Wang, M., Zhao, Y., Yao, Q., Zhang, Q., et al. (2016). Increased default mode network connectivity and increased regional homogeneity in migraineurs without aura. J. Headache Pain 17:98. doi: 10.1186/s10194-0160692-z

Zhang, J., Su, J., Wang, M., Zhao, Y., Zhang, Q. T., Yao, Q., et al. (2017a). The posterior insula shows disrupted brain functional connectivity in female migraineurs without aura based on brainnetome atlas. Sci. Rep. 7:16868. doi: 10.1038/s41598-017-17069-8

Zhang, J., Su, J., Wang, M., Zhao, Y., Zhang, Q. T., Yao, Q., et al. (2017b). The sensorimotor network dysfunction in migraineurs without aura: a resting-state fMRI study. J. Neurol. 264, 654-663. doi: 10.1007/s00415-017-8404-4

Conflict of Interest: The authors declare that the research was conducted in the absence of any commercial or financial relationships that could be construed as a potential conflict of interest.

Publisher's Note: All claims expressed in this article are solely those of the authors and do not necessarily represent those of their affiliated organizations, or those of the publisher, the editors and the reviewers. Any product that may be evaluated in this article, or claim that may be made by its manufacturer, is not guaranteed or endorsed by the publisher.

Copyright (C) 2022 Qin, Liang, Li, Hu, Wu, Qiao, Liu and Du. This is an openaccess article distributed under the terms of the Creative Commons Attribution License (CC BY). The use, distribution or reproduction in other forums is permitted, provided the original author(s) and the copyright owner(s) are credited and that the original publication in this journal is cited, in accordance with accepted academic practice. No use, distribution or reproduction is permitted which does not comply with these terms. 